\title{
Genetic characterization of antithrombin, protein $C$, and protein $S$ deficiencies in Polish patients
}

\author{
Ewa Wypasek ${ }^{1,2}$, Javier Corral ${ }^{3}$, Martine Alhenc-Gelas ${ }^{4}$, Wojciech Sydor ${ }^{5}$, \\ Teresa Iwaniec ${ }^{5}$, Magdalena Celińska-Lowenhoff ${ }^{5}$, Daniel P. Potaczek ${ }^{1,6}$, Aleksandra \\ Blecharczyk ${ }^{1}$, Krystyna Zawilska ${ }^{7}$, Jacek Musial ${ }^{5}$, Anetta Undas ${ }^{1,2}$ \\ 1 John Paul II Hospital, Kraków, Poland \\ 2 Institute of Cardiology, Jagiellonian University Medical College, Kraków, Poland \\ Centro Regional de Hemodonación, Universidad de Murcia, CIBERER, Murcia, Spain \\ Hématologie Biologique, AP-HP Hôpital Européen G. Pompidou, Paris, France \\ 5 Department of Internal Medicine, Jagiellonian University Medical College, Kraków, Poland \\ 6 Institute of Laboratory Medicine and Pathobiochemistry, Molecular Diagnostics, Philipps-Universität Marburg, Marburg, Germany \\ 7 Diagnostic and Treatment Centre INTERLAB, Poznan University of Medical Sciences, Poznań, Poland
}

\section{KEY WORDS}

antithrombin, protein C, protein $\mathrm{S}$, Polish patients, venous thromboembolism
Correspondence to: Ewa Wypasek, PhD, Instytut Kardiologii, Uniwersytet Jagielloński, Collegium Medicum, ul. Prądnicka 80, 31-202 Kraków, Poland, phone: +48 1261431 45, e-mail: ewa.wypasek@uj.edu.pl Received: May 10, 2017. Revision accepted: June 9, 2017. Published online: June 13, 2017. Conflict of interest: none declared. Pol Arch Intern Med. 2017; 127 (7-8): 512-523 doi:10.20452/pamw.4045 Copyright by Medycyna Praktyczna Kraków 2017

\section{ABSTRACT}

INTRODUCTION Inherited deficiencies of natural anticoagulants such as antithrombin (AT; gene: SERPINC1), protein C (PC; $P R O C)$, and protein S (PS; PROS1), with the prevalence in the general European population of $0.02 \%$ to $0.17 \%, 0.2 \%$ to $0.3 \%$, and $0.5 \%$, respectively, are associated with increased risk of thromboembolic events. Only a few case reports of Polish deficient patients with known causal mutations have been published so far.

OBJECTIVES The aim of the study was to characterize the frequency of SERPINC1, PROC, and PROS1 mutations and their thromboembolic manifestations in patients with AT, PC, or PS deficiencies, inhabiting southern Poland.

PATIENTS AND MEETHOdS Ninety unrelated patients (mean [SD] age, 40.1 [13.2] years) with AT ( $n=35$ ), PC $(n=28)$, or PS $(n=27)$ deficiencies, with a history of venous $73(81 \%)$ or arterial $17(19 \%)$ thromboembolism, were screened for mutations using the Sanger sequencing or multiplex ligation-dependent probe amplification.

RESULTS Twenty mutations (29\%) described here were new, mostly in the SERPINC1 and PROC genes. Missense mutations accounted for $84 \%$ of all mutations in the $P R O C$ gene and approximately $50 \%$ of those in the SERPINC1 and PROS1 genes. In all 3 genes, the ratio of nonsense and splice-site mutations was $8 \%$ to $31 \%$ and $8 \%$ to $23 \%$, respectively. The mutation detection rate was $90 \%$ for AT or PC when anticoagulant activity was below $70 \%$, while for the PROS1 gene, the rate reached $80 \%$ at the free PS levels below $40 \%$.

CONCLUSIONS To our knowledge, this is the largest cohort of Polish patients deficient in natural anticoagulants and evaluated for the causal genetic background. Several new Polish detrimental mutations were detected, mostly in AT- and PC-deficient patients.

INTRODUCTION The deficiencies of natural anticoagulants such as antithrombin (AT), protein $C(P C)$, and protein $S(P S)$ are inherited in most of the cases in an autosomal dominant pattern with variable penetrance. In the general European population, they have a frequency of $0.02 \%$ to $0.2 \%, 0.2 \%$ to $0.3 \%$, and $0.5 \%$, respectively..$^{1-3}$ Individuals with inherited deficiencies of natural anticoagulants have an increased risk of venous thromboembolism (VTE), a disease whose management is still challenging and requires ongoing research and clinical studies. ${ }^{4,5}$ 
A meta-analysis of case-control and cohort studies has shown that AT-deficient individuals have a higher risk of the first VTE (odds ratio [OR], 16.3; 95\% CI, 9.9-26.7) than those with PC (OR, 7.1; 95\% CI, 3.2-17.5) or PS (OR, 5.4; 95\% CI, 2.7-10.7) deficiency. ${ }^{6}$ In patients after the first incident of VTE, the prevalence of AT, PC, and PS deficiencies is estimated at $0.5 \%$ to $4.9 \%, 3 \%$, and $2 \%$ to $12 \%$, respectively. ${ }^{2,3,7}$ Evidence linking natural anticoagulant deficiencies with arterial thromboembolism, mostly ischemic stroke, is conflicting and largely derived from case reports. ${ }^{2,8,9}$

$\mathrm{AT}$ is the main endogenous anticoagulant serpin (serine-protease inhibitor) that primarily inactivates thrombin and activated factor $(\mathrm{F}) \mathrm{X}$. The gene encoding AT (SERPINC1) is located on chromosome 1q25.1 and consists of 7 exons and 6 introns spanning 13,574 bp (ENSG00 000117601). ${ }^{10}$ PC and PS are vitamin K-dependent glycoproteins. PC is a precursor of the serine protease, activated protein $C$ (APC). Its proteolytic activation by thrombin occurs on the surface of endothelial cells and involves thrombomodulin and endothelial PC receptor (EPCR). In the presence of PS, phospholipids, and calcium, APC inactivates activated FV and FVIII by their cleavage at the specific arginine residues. ${ }^{11}$ Plasma PS is in $60 \%$ noncovalently complexed with the complement 4b-binding protein (C4BP), while the remaining $40 \%$ circulates free. ${ }^{12}$ Both forms of PS serve as cofactors of APC but free PS is more potent. ${ }^{13}$

$P C$ is encoded by $P R O C$, a gene located on the long arm of chromosome 2q14. PROC is approximately $10842 \mathrm{bp}$ long (ENSG00000115718) and comprises 9 exons separated by 8 introns. ${ }^{14}$ The PS gene (PROS1) is in turn located near the centromere of chromosome 3q11.2, spans 101058 bp (ENSR00 001368680), and consists of 15 exons and 14 introns. ${ }^{15}$

The mutation profiles of all the 3 genes, SERPINC1, PROC, and PROS1, are similar with the highest prevalence for missense mutations (63\%-78\%), followed by nonsense mutations (7\%-11\%), splice-site mutations $(7 \%-13 \%)$, small deletions (1\%-8\%), small insertions/duplications $(1 \%-4 \%)$, and large deletions $(3 \%-6 \%) .{ }^{16}$

Thus far, the genetic deficiency of AT has been reported in 18 Polish families from Warsaw. ${ }^{17}$ To the best of our knowledge, we report here on the largest cohort of patients from Poland, the country with over 38 million inhabitants, with the deficiency of natural anticoagulants, evaluated for the causal genetic background. Previously, we were the first to publish individual Polish cases genetically confirmed for $\mathrm{AT},{ }^{18} \mathrm{PC},{ }^{19}$ and $\mathrm{PS}^{20}$ deficiencies.

PATIENTS AND METHODS Patients A total of 100 unrelated patients with a personal history of thromboembolic events ( $n=89$ ), positive family history $(n=4)$, or obstetric complications $(n=7)$ in association with reduced AT, PC, or PS levels were enrolled in the current study between
January 2013 and December 2016. Of the initially enrolled subjects, 10 were ineligible owing to incomplete clinical and laboratory data $(\mathrm{n}=7)$ or patient refusal $(\mathrm{n}=3)$; thus, $90 \mathrm{pa}$ tients with AT ( $\mathrm{n}=35)$, PC $(\mathrm{n}=28)$, and PS ( = 27) deficiencies were available for the purpose of the present study. All patients were inhabitants of southern Poland, mainly the Lesser Poland (Małopolska) region.

The diagnosis of deep vein thrombosis (DVT) was established on the basis of a positive finding of color duplex sonography (the visualization of an intraluminal thrombus in the calf, popliteal, femoral, or iliac vein). The diagnosis of pulmonary embolism (PE) was based on the presence of typical symptoms and positive results of computed tomography (CT) pulmonary angiography.

A VTE episode was defined as unprovoked (idiopathic) if the patient had no history of cancer, surgery requiring general anesthesia, major trauma, a plaster cast or hospitalization within the last month, or pregnancy or delivery within the last 3 months. A proximal DVT was defined as thrombosis in the popliteal veins, including the trifurcation, femoral, or iliac veins.

The diagnosis of ischemic stroke was based on clinical symptoms according to the World Health Organization definition (http://www. who.int/medicines/areas/priority_medicines/ BP6_6Stroke.pdf?ua=1) and brain imaging, usually noncontrast $\mathrm{CT}$. The diagnosis of myocardial infarction was based on the 2012 European Society of Cardiology criteria. ${ }^{21}$

The diagnosis of portal, splenic, and subclavian vein thrombosis was based on positive findings on a color duplex scan. In more complicated cases, CT angiography or magnetic resonance imaging was needed. The diagnosis of central retinal vein occlusion was based on typical clinical symptoms (abrupt unilateral vision deterioration) and typical appearance of the eye fundus.

The diagnosis of femoral and brachial artery thrombosis was established on the basis of clinical symptoms of acute extremity ischemia and visualization of artery stenosis by color duplex scan sonography or by digital subtraction ultrasound. The diagnosis of mesenteric artery thrombosis was based on clinical symptoms of acute intestinal ischemia and positive results of $\mathrm{CT}$ angiography. The diagnosis of cerebral venous sinus thrombosis was established by visualizing sinus stenosis on magnetic resonance angiography.

The exclusion criteria were previous or current diagnosis of liver disease, cancer, pregnancy, and blood sampling at acute thrombotic event.

The Ethical Committee of Jagiellonian University Medical College approved the study, and all 90 participants provided their written informed consent.

Sample collection Blood samples were drawn from an antecubital vein into tubes containing citrate anticoagulant (9:1 of $0.106 \mathrm{M}$ sodium citrate), centrifuged at $2.500 \mathrm{~g}$ at a temperature of 
$18^{\circ} \mathrm{C}$ to $22^{\circ} \mathrm{C}$ for 20 minutes and processed immediately or stored in aliquots at $-80^{\circ} \mathrm{C}$ until analysis. Whole blood samples for DNA isolation were drawn into EDTA-K3 collection tubes and stored in aliquots at $-80^{\circ} \mathrm{C}$ until processing.

Laboratory tests The antigen levels and activities of AT, PC, and PS were measured using established commercial assays after temporary withdrawal of vitamin $\mathrm{K}$ antagonists (for at least 10 days) ${ }^{22}$ or oral novel anticoagulants (for at least 24 hours). ${ }^{23}$

Antithrombin AT activity was measured using an assay based on FXa inhibition (INNOVANCE ${ }^{\mathrm{TM}}$ ATIII, Siemens Healthcare Diagnostics, Marburg, Germany) or thrombin-inhibition assays (Siemens Healthcare Diagnostics); the reference range for both was from $83 \%$ to $118 \%$. AT antigen was determined nephelometrically (Siemens Healthcare Diagnostics; reference range, $0.19-0.31 \mathrm{~g} / \mathrm{l})$. AT deficiency was classified as described previously. ${ }^{10,24}$

Protein C Plasma PC activity was quantified using a chromogenic assay (HemosIL Protein C Instrumentation Laboratory, Milan, Italy or Berichrom Protein C, Siemens Healthcare Diagnostics), and PC antigen was measured by an enzyme-linked immunosorbent assay (Asserachrom Protein C, Diagnostica Stago, Asnieres, France) (reference range for all, 70\%-140\%). PC deficiency was classified as described previously. ${ }^{25}$

Protein S Free PS levels were measured using an immunoturbidimetric assay (HemosIL Free Protein S; reference range for women, $55 \%-124 \%$; reference range for men, $74 \%-146 \%$ or INNOVANCE ${ }^{\circledR}$ Free PS Ag, Siemens Healthcare Diagnostic; reference range for women, $60 \%-114 \%$; reference range for men, $67 \%-139 \%)$. Total PS levels were assessed using Asserachrom Kit (Diagnostica Stago; reference range, $75 \%-140 \%$ ). PS deficiency was classified as previously described. ${ }^{25}$

Genetic analysis DNA was extracted from whole blood or a buffy coat according to the manufacturer's protocol, using Gene MATRIX Quick Blood DNA Purification Kit (Eurex, Gdańsk, Poland) and stored at $-80^{\circ} \mathrm{C}$ until analysis.

The SERPINC1 (NM_000488.3), PROC (NM_000 312.3), and PROS1 (NM_000 313.3) genes were analyzed as previously described. ${ }^{26-28}$ For all samples with no causative point mutation identified, screening for large deletion/insertion was performed by multiplex ligation-dependent probe amplification (SALSA MLPA kit for P227 SerpinC1; P265-PROC and P112-A2 PROS1; MRC-Holland, Amsterdam, the Netherlands).

The analysis of a congenital disorder of glycosylation was performed as described previously. ${ }^{29}$

In silico analysis Each novel sequence variation was subjected to in silico analyses with a Sorting
Intolerant From Tolerant tool (http://sift.jcvi.org) integrated into Alamut Visual v2.3.6 software application (http://www.interactive-biosoftware. com/alamut-visual/); a structure-based method, Polymorphism Phenotyping v2 (PolyPhen-2, http://genetics.bwh.harvard.edu/pph); and the splicing prediction module of Alamut Visual, which uses the Human Splicing Finder v3.0 (HSF; http://www.umd.be/HSF/) algorithms.

Statistical analysis The distributions of quantitative variables were analyzed by the Shapiro-Wilk test. Normally distributed variables were compared using 1-way analysis of variance (ANOVA) or the $t$ test and were presented as mean (SD). Variables deviating from normal distribution were analyzed by the Kruskal-Wallis ANOVA or Mann-Whitney test and were presented as median (interquartile range) if not otherwise indicated. Qualitative parameters were analyzed by the Pearson $\chi^{2}$ or 2-tailed Fisher exact test. Pairwise correlations were calculated using the Pearson coefficient on variables approximated to the normal distribution if required. A $P$ value of less than 0.05 was considered significant. Statistical calculations were performed using STATISTICA Version 10.0 (StatSoft, Inc., Tulsa, Oklahoma, United States).

RESULTS The mean (SD) age of 90 participants with natural anticoagulant deficiencies was 40.1 (13.2) years. Forty-seven patients (52.2\%) were male. The mean (SD) age at the first VTE event was 35.6 (12.4) years. Thirty-one patients (34\%) experienced a provoked VTE event, mostly despite pharmacological thromboprophylaxis. These patients were younger than those with an unprovoked VTE (mean [SD] age, 32.2 [2.2] years vs 44.3 [1.8] years; $P=0.02$ ). Thirty-four patients (38\%) had a positive family history of VTE.

Of the 90 patients enrolled, 65 (72\%) carried genetic defects. Twenty mutations (29\%) detected in the current study have been reported for the first time, according to the comparison with the $\mathrm{HGMD}^{\circledR}$ database records. All new mutations were probably damaging and disease-causing as suggested by the in silico analysis.

Antithrombin deficiency The main characteristics of the AT-deficient cohort $(\mathrm{n}=35)$ are shown in tABLE 1. The mean (SD) age was 43.0 (13.9) years, and 20 patients $(57 \%)$ were male. The mean (SD) age at the first VTE event was 37.1 (14.1) years. The mean (SD) AT activity was $57.8 \%$ (9.3\%) (less than $75 \%$ in all participants), and the median (IQR) AT antigen level was $0.16 \mathrm{~g} / \mathrm{l}(0.15-0.22 \mathrm{~g} / \mathrm{l})$. Type I AT deficiency was detected in 19 patients (54.3\%) and type II-in 11 patients (31.4\%). In the remaining 5 patients (14.3\%), the type of AT deficiency could not be determined due to incomplete laboratory data.

Antigen levels correlated with age of the first VTE event $(r=0.6 ; P=0.002)$. Patients with type I AT deficiency were younger when they had experienced the first VTE event compared with patients 


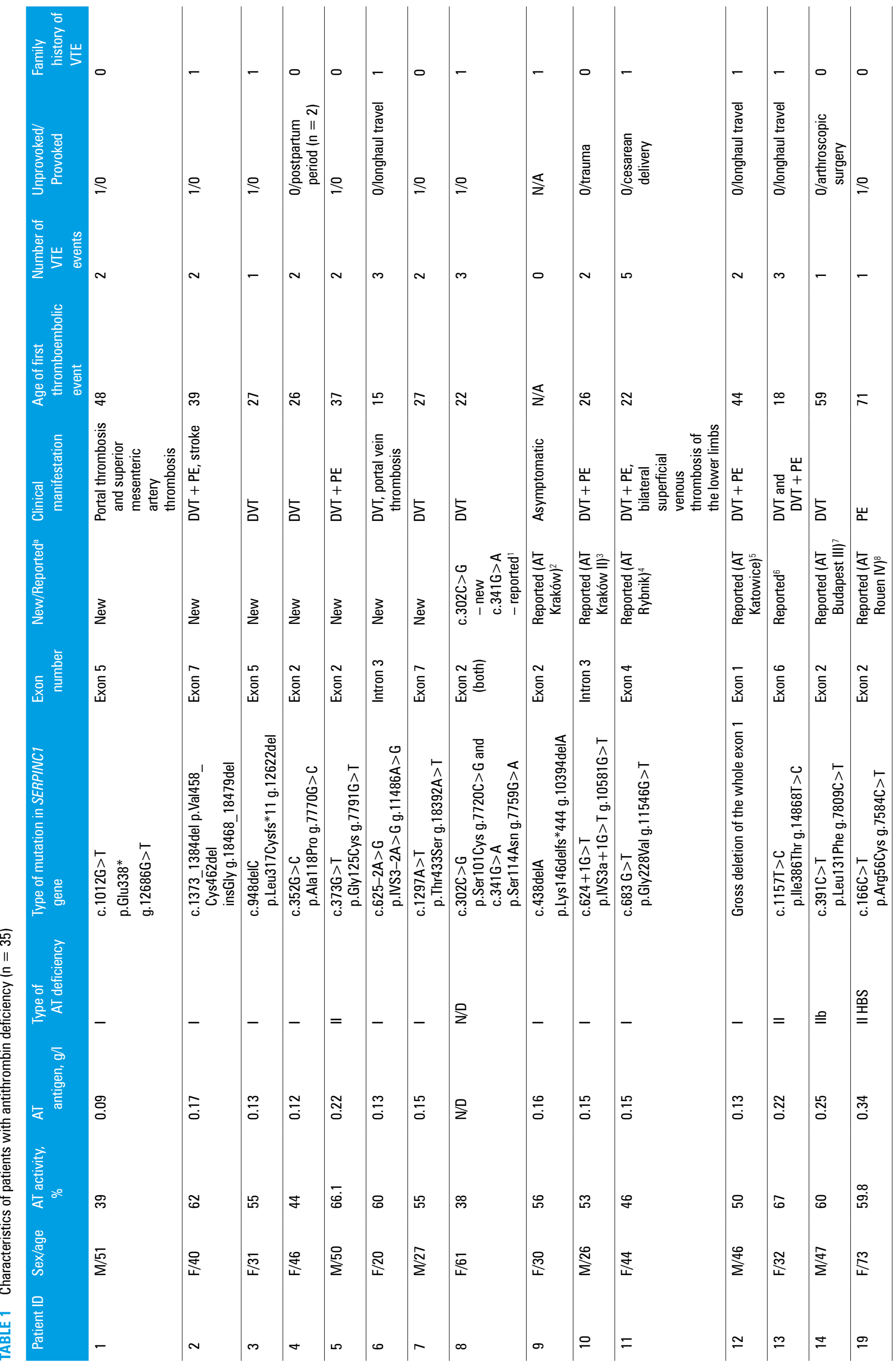




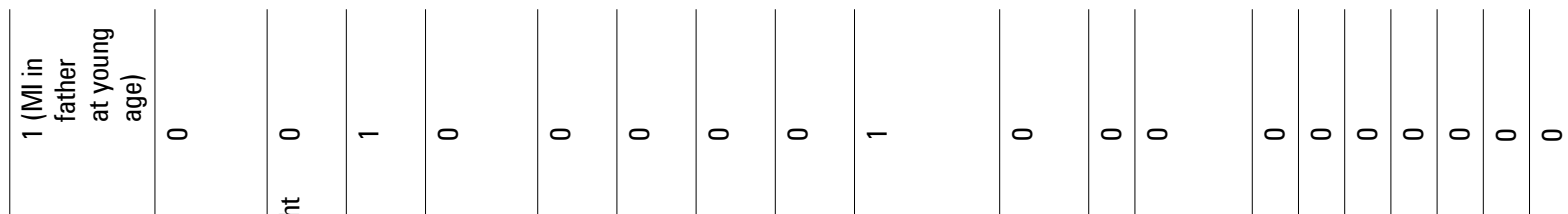

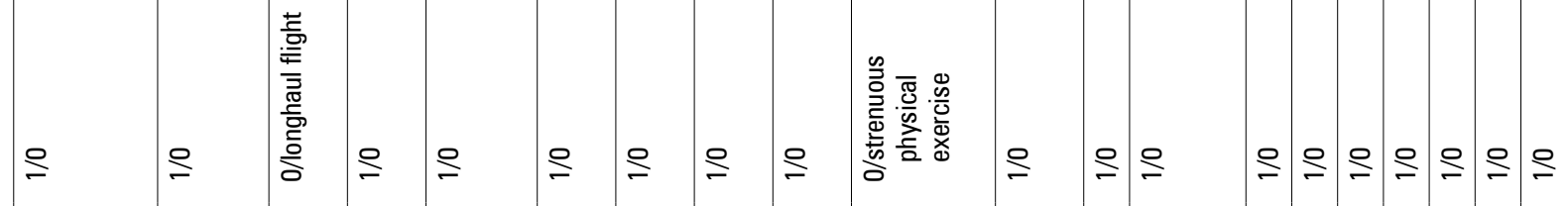

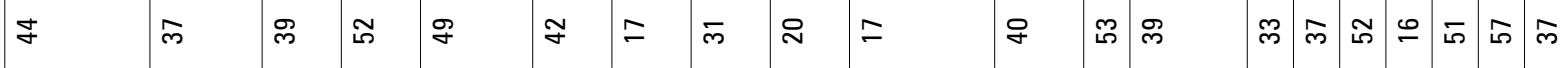

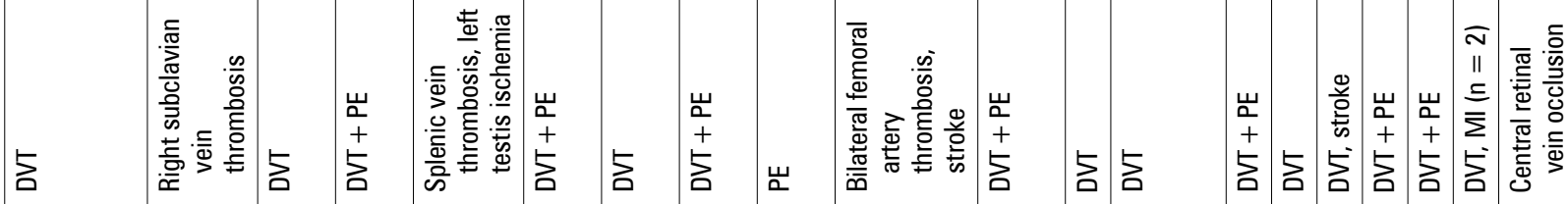

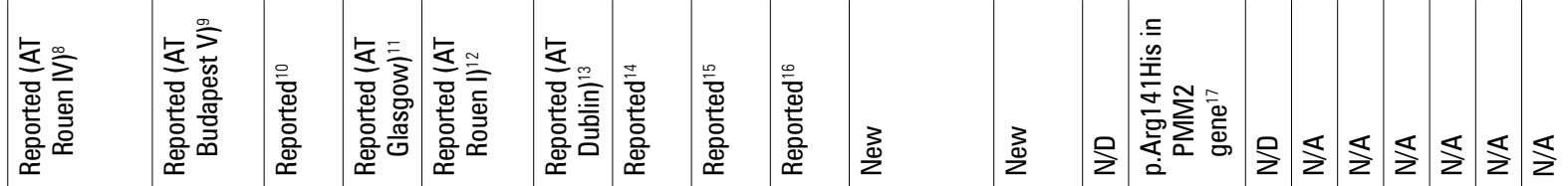

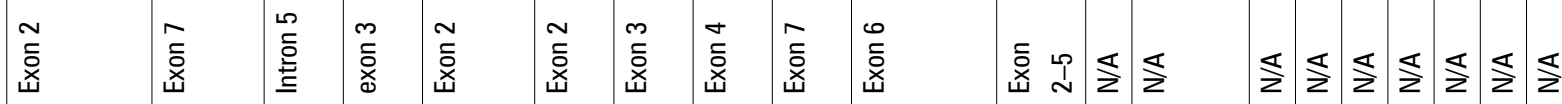

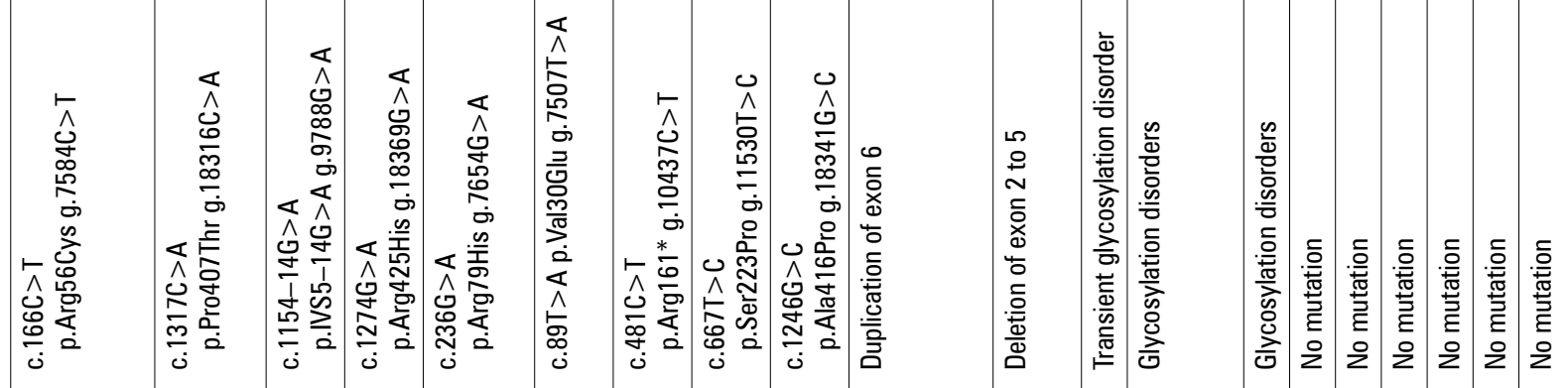

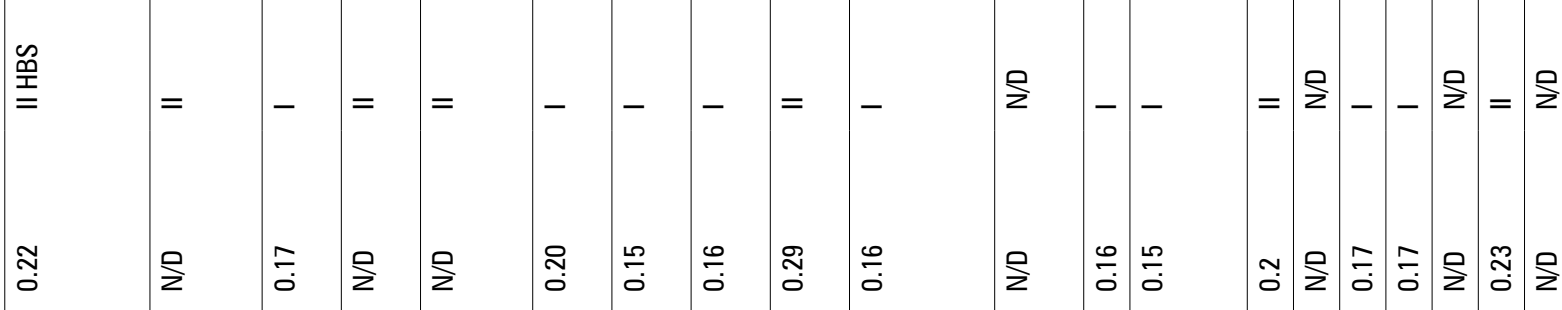

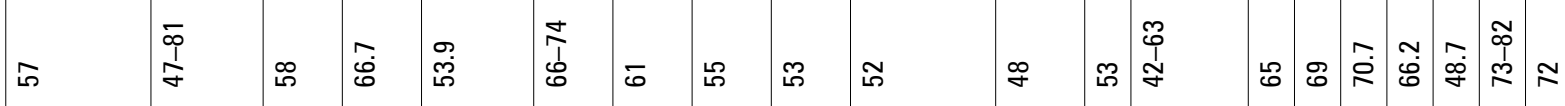

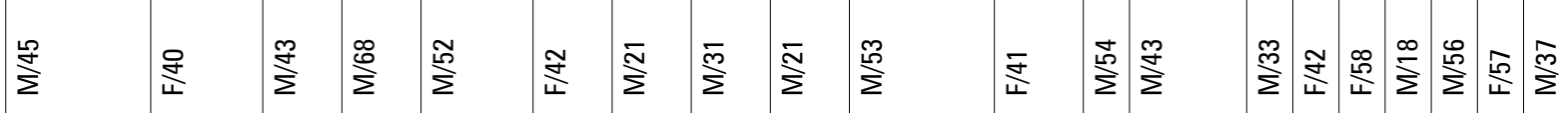

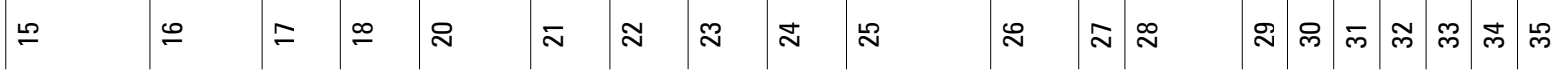


with type II AT deficiency (mean [SD] age, 32.2 [3.2] years vs 45.7 [4.3] years; $P=0.02$ ).

SERPINC1 mutations Of the 35 patients with AT deficiency, 26 individuals had a mutation in the SERPINC1 gene, with a mutation detection rate of $74 \%$. Glycosylation disorders with mutation in the phosphomannomutase 2 gene (PMM2) were detected in 3 patients.

Missense mutations represented the most frequent causal genetic variant type and were identified in 15 patients (57.7\%), followed by nonsense mutations in 4 patients (15.4\%), splice-site mutations in 3 patients (11.5\%), large deletions in 2 patients $(7.8 \%)$, and small deletion and large duplication (1 patient [3.8\%] each). In half of the patients (50\%), mutations were located mainly in exon 2 (9 patients) or exon 7 (4 patients).

Eight (31\%) mutations were reported here for the first time (TABLE 1). The whole spectrum of mutations was present in patients with type I AT deficiency, while in patients with type II AT deficiency, only the missense variants were found. Patients with missense mutations tended to have higher AT antigen expression than individuals with other types of causal genetic variants $(0.21 \mathrm{~g} / 1$ vs $0.15 \mathrm{~g} / \mathrm{l}, P=0.06)$.

Venous and arterial thrombosis The most common thrombotic manifestation was isolated DVT ( $\mathrm{n}=14 ; 40 \%)$ or combined with PE $(\mathrm{n}=13$; $37 \%$ ). Isolated $\mathrm{PE}$ was present only in 2 patients. Moreover, we observed patients with portal vein thrombosis $(n=2 ; 6 \%)$, subclavian vein thrombosis $(n=1 ; 3 \%)$, splenic vein thrombosis and testis ischemia ( $n=1 ; 3 \%)$, and central retinal vein occlusion ( $\mathrm{n}=1 ; 3 \%)$ (TABLE 1$)$.

Arterial thromboembolic events occurred in 5 patients (14\%) and comprised 3 cerebral ischemic strokes, 1 myocardial infarction (with recurrence), and 1 superior mesenteric artery thrombosis combined with portal vein thrombosis. One individual was asymptomatic but had a positive family history.

Protein C deficiency The main characteristics of the PC-deficient patients are shown in TABLE 2. The mean (SD) age of the patients was 38.3 (13.4) years. Sixteen patients (57\%) were male. The first VTE event occurred at a mean (SD) age of 35.0 (12.6) years.

The mean (SD) PC activity was $51.1 \%$ (12.9\%), and the mean (SD) total PC was $63.4 \%$ (25.9\%). Thirteen patients $(46.4 \%)$ had type I PC deficiency, and 2 individuals (7.2\%) - type II. In 13 patients (46.4\%), the type of PC deficiency could not be determined owing to incomplete laboratory data.

As expected, PC activity was positively correlated with age $(r=0.38 ; P<0.05)$. Patients who experienced an idiopathic VTE were older than those with a provoked VTE (mean [SD] age, 40.8 [3.4] years vs 30.8 [3.6] years; $P=0.05$ ) and had higher PC activity levels (mean [SD], 57.9\% [3.5\%] vs $42.8 \%$ [3.7\%]; $P=0.008)$.
PROC mutations Of the 28 patients with PC deficiency, 25 individuals had the mutation in the $P R O C$ gene, with a mutation detection rate of $89.3 \%$.

Missense mutations occurred in 21 patients (84\%), while nonsense and splice-site mutations in 2 patients each ( $8 \%$ for both). No gross $P R O C$ gene rearrangements were observed.

The detected mutations were mainly located in exon 9 (9 patients), exon 5 (5 patients), and in exon 7 (4 patients), which comprised $72 \%$ of the patients. Eight mutations (32\%) have been reported here for the first time (TABLE 2).

Venous and arterial thrombosis The most common thrombotic manifestation was isolated DVT ( $n=10 ; 36 \%)$ or combined with PE ( $n=9 ; 32 \%)$. Isolated PE was not found. One patient (3.6\%) suffered from DVT and superficial vein thrombosis. Among atypical VTE, portal vein thrombosis ( $n=1 ; 3.6 \%)$ and cerebral venous sinus thrombosis $(n=1 ; 3.6 \%)$ were observed. One woman experienced 3 miscarriages (3.6\%).

Arterial thrombosis occurred in 5 patients (18\%) and included 2 myocardial infarctions, 2 strokes, and brachial arterial embolism. Three subjects were asymptomatic but had a positive family history (TABLE 2).

Protein S deficiency The main characteristics of the PS-deficient cohort are shown in TABLE 3. The mean (SD) patient age was 38.2 (13.1) years. Ten patients (37\%) were male. The first VTE event occurred at the mean (SD) age of 34.0 (9.4) years.

The mean (SD) free PS levels were $37.0 \%$ (14.7\%) and the mean (SD) total PS antigen was $62.7 \%$ (18.0\%). Fourteen patients $(52.5 \%)$ had type I and 11 patients (40.1\%) had type III PS deficiency. In 2 patients (7.4\%), the type of PS deficiency could not be determined owing to in complete laboratory data or variations in total PS levels.

Patients with idiopathic VTE had lower free PS levels than those with provoked VTE (mean [SD], $27.1 \%$ [4.0\%] vs $41.6 \%$ [4.0\%]; $P=0.02$ ).

PROS1 mutations Of the 27 patients with PROS1 deficiency, 14 individuals had the PROS1 gene mutation with a mutation detection rate of $52 \%$. If the cutoff value for free PS levels was above $40 \%$, the mutation detection rate reached $77 \%$.

Missense mutations were observed in $6 \mathrm{pa}$ tients (46\%); nonsense mutations, in 4 patients (31\%); and splice-site mutations, in 3 patients (23\%). One deletion of exons 1 to 12 was found. The detected mutations were located across the whole PROS1 gene. Three mutations (21\%) have been reported here for the first time (TABLE 3).

Venous and arterial thrombosis The most common thrombotic manifestation was isolated DVT ( $\mathrm{n}=15 ; 55.7 \%$ ). DVT combined with PE was observed in 3 patients (11.1\%), and isolated $\mathrm{PE}$-in 1 patient (3.7\%). One event of cerebral venous 
sinus thrombosis (3.7\%) and four miscarriages (14.8\%) were noted.

Arterial thrombosis was found in 7 patients (26\%), including 7 strokes, 2 of which occurred after myocardial infarction (TABLE 3).

Mutation detection rate AT activity levels ranged between $38 \%$ and $67 \%$ in patients with a detected SERPINC1 gene mutation and glycosylation disorders, and between $49 \%$ and $77.5 \%$ in subjects without the mutation. In individuals with AT activity above $70 \%$, no mutations were found, while in those with AT activity below $70 \%$, the mutation detection rate was $90 \%$.

Similarly, patients with a mutation in the PROC gene showed PC activity between $19 \%$ and $67 \%$. In 3 patients without the mutation, PC activity levels ranged from $18 \%$ to $53 \%$. For PC activities close to the normal range $(70 \%)$, the mutation detection rate was above $90 \%$.

Patients with a mutation within the PROS1 gene had the PS activity levels of $8 \%$ to $53 \%$, and those without the mutation-of $37 \%$ to $59 \%$. For free PS levels below $40 \%$, the mutation detection rate was $77 \%$, and for free PS levels below $35 \%$, the rate was $100 \%$.

DISCUSSION To the best of our knowledge, this is the first large and comprehensive study analyzing the genetic background of deficiencies in natural anticoagulants, AT, PC, and PS, not only in the Polish but also in the Slavic population. Twenty novel mutations were identified, all of which present in the heterozygous state.

The frequency of missense, nonsense, and splice-site mutations was similar for all 3 genes/ proteins. Missense mutations accounted for approximately $50 \%$ of all the causal variants detected. Large rearrangements were found in the SERPINC1 and PROS1 genes. The detected mutations were located mainly in exon 2 and 7 of the SERPINC1 gene, in exons 9, 7, and 5 of the $P R O C$ gene, and, with no exon-wise clustering, across the whole PROS1 gene.

The newly detected p.Glu338* and the p.Val458_ Cys462del insGly mutations in the SERPINC1 gene might impair protein synthesis/secretion and are most likely responsible for type I AT deficiency. Both variants were found also in the families of the probands and were associated with VTE events. Similarly, another new variant, deletion of cytosine (c.948delC), may lead to production of truncated protein followed by a frameshift and the appearance of a premature stop codon in the SERPINC1 gene. In turn, the new p.Ala118Pro and the p.Gly125Cys variants both affect a very highly conserved residues in serpins located at helix B and may thus probably disturb the correct folding of AT. They may lead to the polymerization of the variant AT molecules and formation of disulphide-linked dimers present in the patient's plasma. These dimers may accumulate intracellularly in hepatocytes, affecting the synthesis of a wild-type AT and other inflammatory or hemostatic proteins, especially in situations associated with increased thrombotic risk, such as pregnancy or infection. ${ }^{30,31}$ In the current study, only a patient carrying the p.Ala118Pro variant had 2 DVT events in the postpartum period. The proband's daughter inherited this mutation and so far remains asymptomatic.

Another new variant is an intronic c.625-2A>G mutation affecting splicing by disturbing the acceptor sequence of intron 3 . This mutation is associated in our patient with type I AT deficiency and was also detected in the proband's father, suffering from DVT. In turn, the next novel variant, the p.Thr433Ser mutation, affects a residue which, although not conserved in serpins, is located at the first strand of $C \beta$-sheet and may thus affect protein stability.

We also detected 1 compound heterozygote within the SERPINC1 gene comprising the novel p.Ser69Cys variant and previously described as p.Ser82Asn mutation. ${ }^{32}$ The p.Ser69Cys variant affects helix A, potentially influencing protein stability. However, the p.Ser82Asn mutation, responsible for creating a new $\mathrm{N}$-glycosylation site associated with disturbances in peptide folding ultimately leading to no protein secretion, ${ }^{32}$ seems to play a more important role. In patients in whom no point mutations were found, 2 new large gene rearrangements were detected: a duplication of exon 6 and a deletion of exons 2 to 5 .

The majority of the newly detected PROC gene mutations, including p.Cys387Tyr, p.Val434Ala, and p.Leu320Pro, cluster in exon 9, within the region encoding a catalytic domain. These variants may potentially affect binding of the substrate. ${ }^{33}$ Moreover, in the missense variant at position 387, cysteine is replaced by tyrosine, which may be important for disulfide bond formation, associated with protein stabilization. In turn, the p.Cys64Tyr variant of the PROC gene is located in the $\gamma$-carboxyglutamic domain within the PC structure. This domain is implicated in interactions of PC with its cofactors PS and EPCR for inactivation of FVa and FVIIIa. ${ }^{34}$ The $\mathrm{p}$. Cys64Tyr variant eliminates cysteine residues, which might result in aberrant protein multimerization or creation of the high-molecular-weight protein complex and impaired protein secretion. ${ }^{33,35}$ A similar mutation in the same codon but with a different amino acid change (p.Cys64Gly) has been reported by Kuismanen et $\mathrm{al}^{35}$ in a family from Finland, with a history of thrombosis and type I PC deficiency. The next new p.Cys175Arg variant within the PROC gene is located in the epidermal growth factor (EGF)-2 domain and might affect PC stability. ${ }^{33}$

Another novel mutation, p.Gln226*, is located in the serine-protease domain and may yield a truncated protein product. It has been found that amino acids at position 225-235 of the PC serine-protease domain play an important role in the interaction between the PC and the thrombin-thrombomodulin complex through a calcium-binding mechanism. ${ }^{36}$ Two other missense 


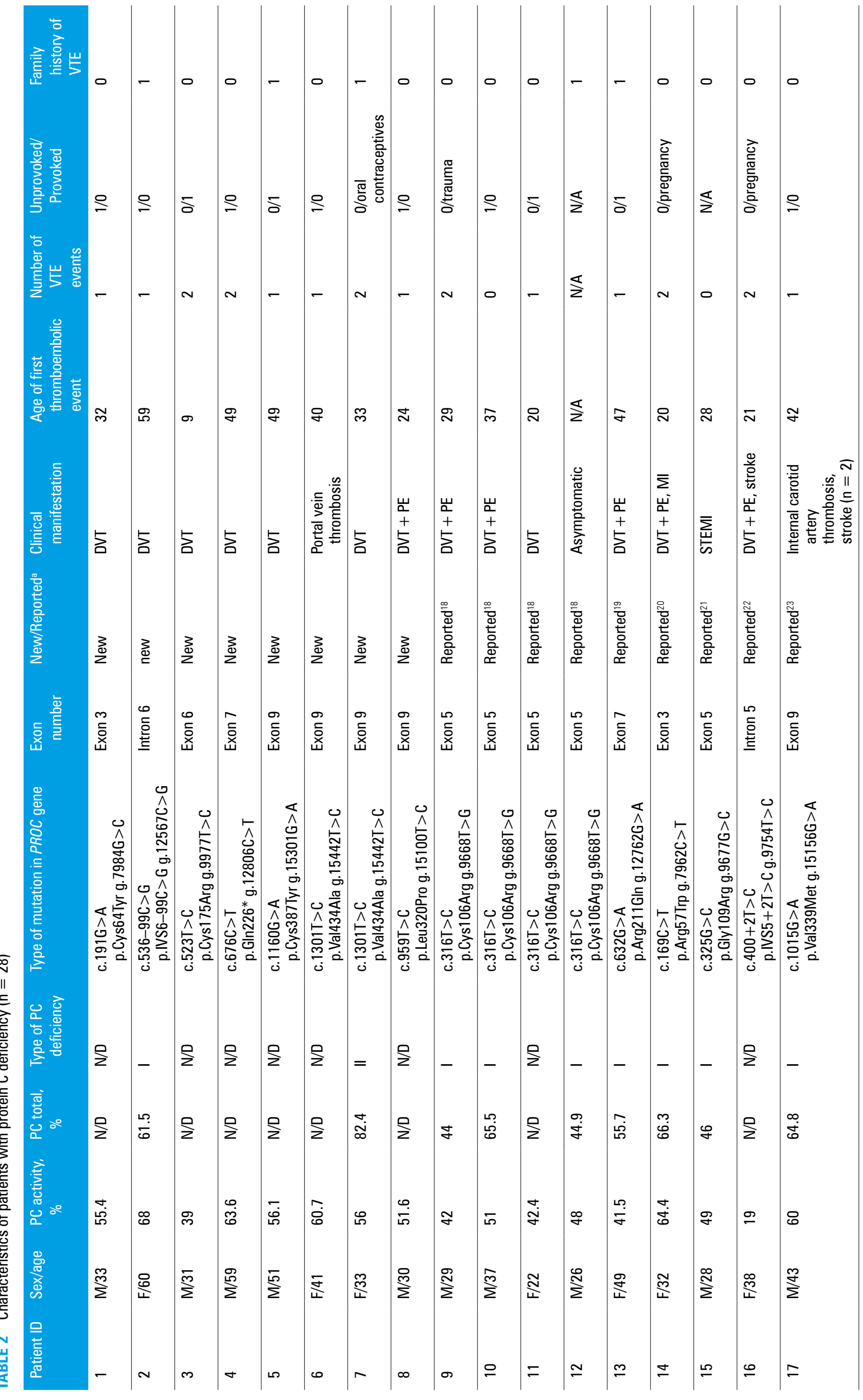




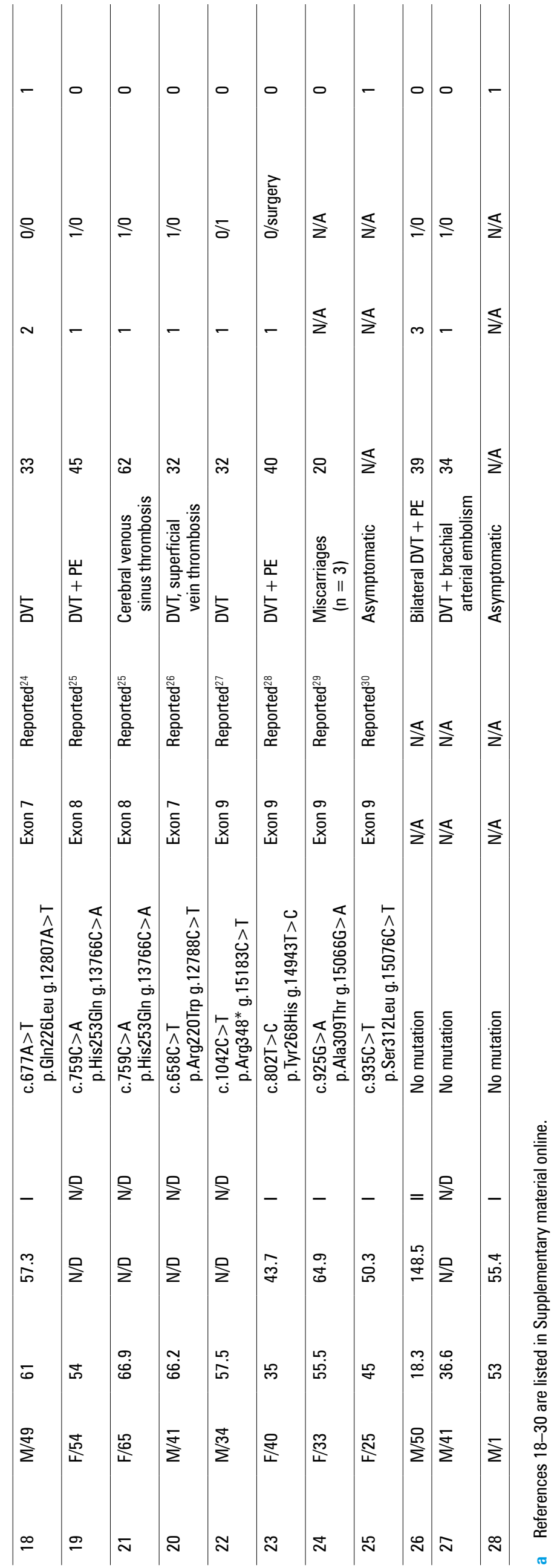

mutations at the same position, p.Gln226His and p.Gln226Leu, have been described in a newborn Spanish child with purpura fulminans and a Spanish patient screened for thrombophilia after a DVT event, respectively. ${ }^{37,38}$ In the current study, the missense variant Gln226Leu in exon 7 was also detected in a 49-year-old male patient with DVT. Interestingly, the p.Cys106Arg variant, previously described by us in a young Polish man with provoked DVT and PE, ${ }^{39}$ was detected in the current study in 3 other unrelated patients.

The 2 new mutations in the PROS1 gene, p.Cys126Gly and the p.Cys241*, are located in the EGF-like- 1 and the EGF-like- 3 calcium binding domains, respectively. It has been shown that most missense mutations identified within the EGF domains of PS affect protein stability, and cysteine residues are replaced in half of them. ${ }^{3}$ It may be speculated that our novel p.Cys126Gly variant disrupts the protein structure, resulting in protein instability. The second newly identified mutation, p.Cys241*, might lead to the production of truncated protein. As PS is a principal cofactor of APC, its impaired secretion would limit the degradation of FVa and FVIIIa and enhance the procoagulant conditions. ${ }^{3,40}$ The last new variant within the PROS1 gene, p.Gly489Arg, is located in the laminin-G-like-2 domain, suggested to comprise the $\mathrm{C} 4 \mathrm{BP}-$ binding sites within segments 408-434, 447-460, and 583-635..$^{41}$ Although this new variant is located outside the reported C4BP-binding sites, changing nonpolar and neutral glycine to polar and basic arginine might be expected to influence the C4BP binding.

In $32 \%$ of AT-deficient, $6 \%$ of PC-deficient, and $50 \%$ of PS-deficient individuals, no mutation in the respective genes was detected in the present study. It is possible that other genes on chromosome $16 q 23$ and 1q32 influence the PC and PS levels. ${ }^{16,42,43}$ PC activity can be modulated also by the genetic variants of EPCR ${ }^{44}$ and the polymorphisms of the genes involved in the vitamin K-dependent $\gamma$-carboxylation reaction may also influence the activities of PC and PS. ${ }^{45}$ It should be also noted that for the measurement of AT activity, both the bovine thrombin- and the FXa inhibition-based tests, together with an antigen method, are advisable to be performed in patients with suspected type II AT deficiency. ${ }^{46}$ For thrombin-based tests, a bovine thrombin should be preferred because a human thrombin reacts also with the heparin cofactor II, thus leading to overestimation of AT. ${ }^{47}$ On the other hand, false negative detection of all types of AT deficiencies with a FXa inhibition-based test, which may overestimate AT activity levels, is also possible.

Interestingly, in the current study, no mutations were found in patients with AT activity above $70 \%$. This is in contrast to a study by Caspers et al, ${ }^{16}$ conducted in German patients, in whom the mutation detection rate remained at a level of about $70 \%$ for AT activities up to $75 \% .{ }^{16}$ It seems that in our population, a genetic analysis of the SERPIC1 gene should be performed in patients 


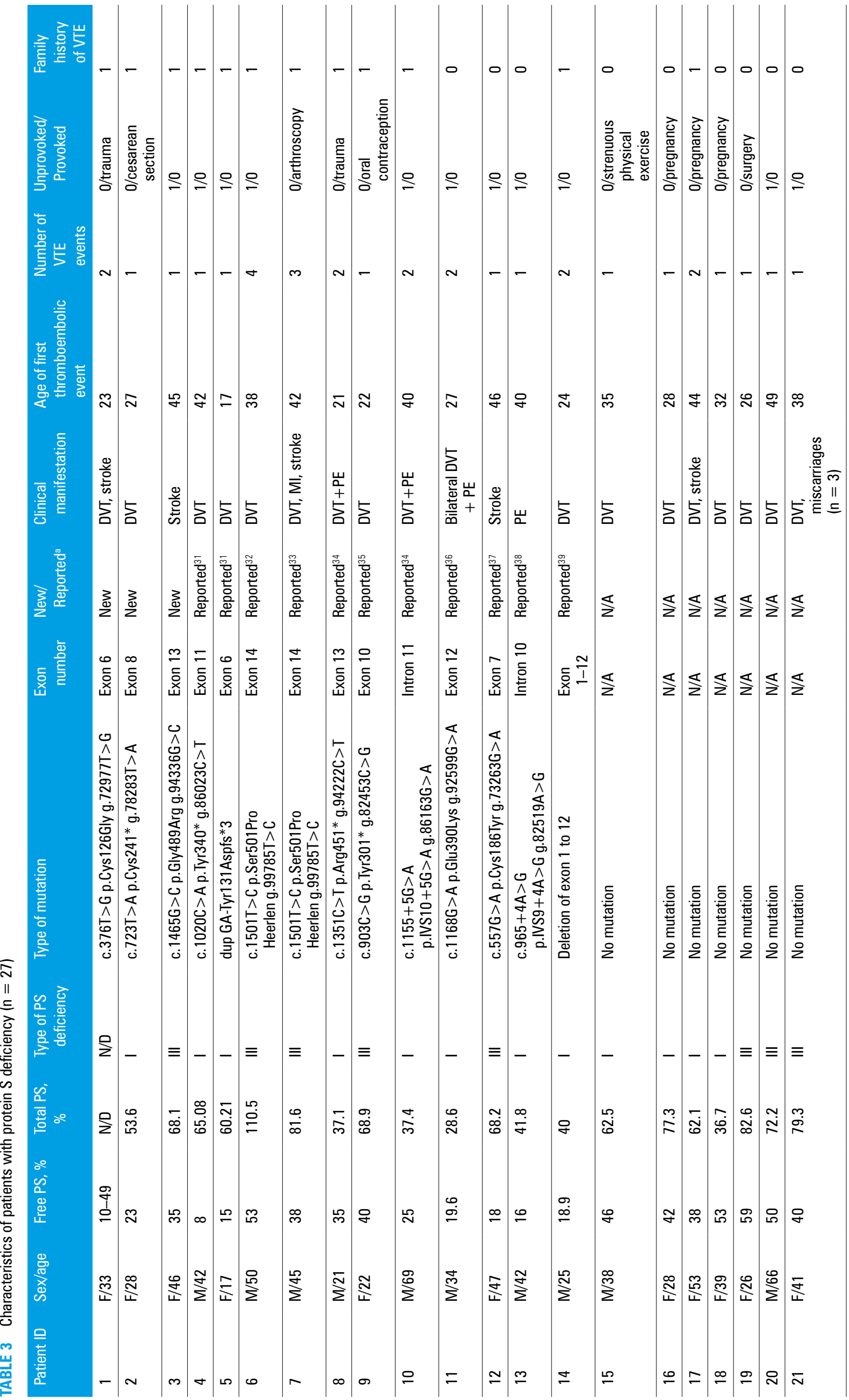




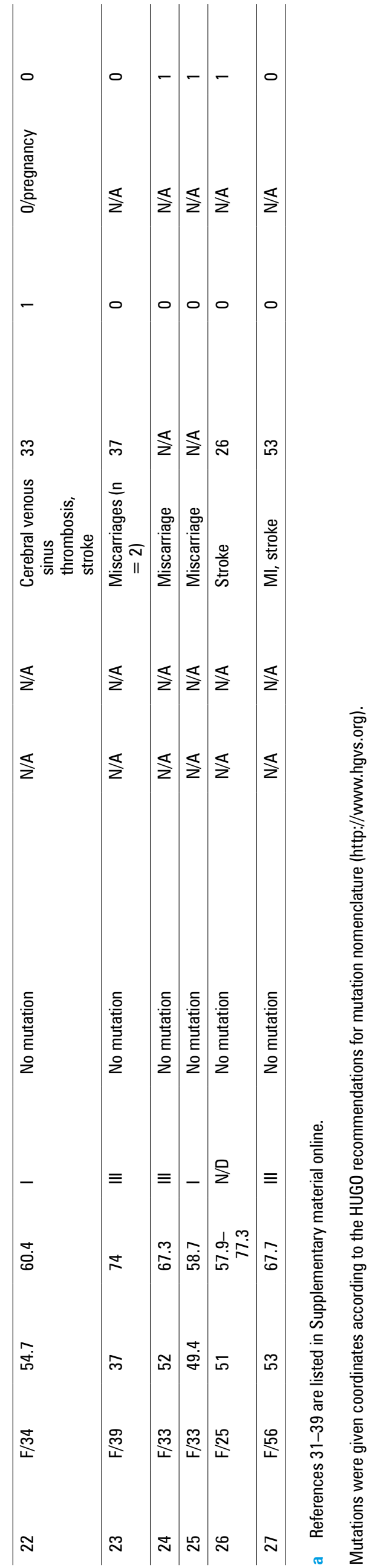

with AT activity below $70 \%$, where the mutation detection rate was $90 \%$. However, due to a small sample size, this finding should be interpreted with caution. On the other hand, the study on the German population showed that the genetic analysis of the $P R O C$ gene should be performed in patients with $\mathrm{PC}$ activities below $70 \%$, which worked fine also for our patients. ${ }^{16}$ Similarly, in our PS-deficient patients, no mutations were detected above $53 \%$ of PS activity, which is in line with the study by Caspers et al. ${ }^{16}$

The major study limitation was a relatively small sample size; however, we analyzed all eligible patients suspected of these types of thrombophilia in our center. For the new mutations, functional studies should be performed in addition to in silico predictions to prove their detrimental effect.

In conclusion, we report here the largest cohort of Polish Slavic patients deficient in natural anticoagulants, whom we evaluated for the causal genetic background. Several new Polish detrimental mutations were detected mostly in patients with AT and PC deficiencies.

Supplementary material online Supplementary material is available with the online version of the article at www.pamw.pl.

Acknowledgments This project was funded by the National Science Centre (2013/09/B/NZ5/00 758; to $\mathrm{AU}$ ) and Jagiellonian University Medical College (K/ZDS/002 936; to AU). We thank Dr. Wiesława Kwiatkowska for patient recruitment.

Contribution statement EW contributed to the study design, interpreted the data, and wrote the manuscript. JC and MA-G performed the laboratory analysis and interpreted the data. WS, MC-L, KZ, and JM recruited patients and interpreted the data. TI performed the laboratory analysis. DPP and AB interpreted the data. AU conceived the study, recruited patients, and gave the final approval.

\section{REFERENCES}

1 Tait RC, Walker ID, Perry DJ, et al. Prevalence of antithrombin deficiency in the healthy population. Br J Haemotol. 1994; 87: 106-112.

2 Whitlatch NL, Ortel TL. Thrombophilias: when should we test and how does it help? Semin Respir Crit Care Med. 2008; 29: 25-39.

3 García de Frutos $\mathrm{P}$, Fuentes-Prior $\mathrm{P}$, Hurtado $\mathrm{B}$, et al. Molecular basis of protein $S$ deficiency. Thromb Haemost. 2007; 98: 543-556.

4 Douketis J, Ageno W, Carrier M, et al. Managing challenging patients with venous thromboembolism: a practical, case-based approach. Pol Arch Intern Med. 2017; 1:41-46.

5 Weitz $\mathrm{Jl}$, Jaffer IH. Optimizing the safety of treatment for venous throm boembolism in the era of direct oral anticoagulants. Pol Arch Med Wewn. 2016; 9: 688-696.

6 Di Minno MN, Ambrosino P, Ageno W, et al. Natural anticoagulants de ficiency and the risk of venous thromboembolism: a meta-analysis of observational studies. Thromb Res. 2015; 5: 923-932.

7 Heit JA. Epidemiology of venous thromboembolism Nat Rev Cardiol. 2015; 8: 464-474.

8 Mahmoodi BK, Brouwer JL, Veeger NJ, van der Meer J. Hereditary deficiency of protein $\mathrm{C}$ or protein $\mathrm{S}$ confers increased risk of arterial thromboembolic events at a young age: results from a large family cohort study. Circulation. 2008; 118: 1659-1667. 
9 Sayin MR, Akpinar I, Karabag T, et al. Left main coronary artery thrombus resulting from combined protein $\mathrm{C}$ and $\mathrm{S}$ deficiency. Intern Med. 2012 51: 3041-3044.

10 Patnaik M, Moll S. Inherited antithrombin deficiency: a review. Haemophilia. 2008; 14: 1229-1239.

11 Dahlbäck B, Villoutreix BO. Molecular recognition in the protein $C$ anticoagulant pathway. J Thromb Haemost. 2003; 1: 1525-1534.

12 Dahlbäck B. Inhibition of protein C cofactor function of human and bovine protein S by C4b-binding protein. J Biol Chem. 1986; 261 12022-12027.

13 Maurissen LFA, Thomassen MC, Nicolaes GAF, et al. Re-evaluation of the role of the protein $\mathrm{S}-\mathrm{C} 4 \mathrm{~b}$ binding protein complex in activated protein C-catalyzed factor Va-inactivation. Blood. 2008; 111: 3034-3041.

14 Foster DC, Yoshitake S, Davie EW. The nucleotide sequence of the gene for human protein C. Proc Natl Acad Sci U S A. 1985; 82: 4673-4677

15 Schmidel DK, Tatro AV, Phelps LG. Organization of the human protein S genes. Biochemistry. 1990; 29: 7845-7852.

16 Caspers M, Pavlova A, Driesen J, et al. Deficiencies of antithrombin protein $\mathrm{C}$ and protein $\mathrm{S}$ - practical experience in genetic analysis of a large patient cohort. Thromb Haemost. 2012; 2: 247-257.

17 Odnoczko E, Vertun-Baranowska B, Buczma A, et al. [Genetic analysis of inherited antithrombin deficiency in 18 Polish families]. Acta Haematol Pol. 2011; 42: 519-524. Polish.

18 Celinska-Löwenhoff M, Iwaniec T, Alhenc-Gelas M, et al. Arterial and venous thrombosis and prothrombotic fibrin clot phenotype in a Polish family with type 1 antithrombin deficiency (antithrombin Krakow). Thromb Haemost. 2011; 2: 379-381.

19 Wypasek E, Pankiw-Bembenek 0, Potaczek DP, et al. A missense mutation G109R in the PROC gene associated with type I protein C deficiency in a young Polish man with acute myocardial infarction. Int $\mathrm{J}$ Cardiol. 2013; 5: 46-48.

20 Wypasek E, Alhenc-Gelas M, Undas A. First report of a large PROS deletion from exon 1 through 12 detected in Polish patients with deep-vein thrombosis. Thromb Res. 2013; 1: 143-144.

21 Taylor J. Third universal definition of myocardial infarction. Eur Heart J. 2012; 20: 2506-2507.

22 Wypasek E, Undas A. Protein C and protein S deficiency - practical diagnostic issues. Adv Clin Exp Med. 2013; 4: 459-467.

23 Undas A, Góralczyk T. Direct oral anticoagulants in patients with thrombophilia: challenges in diagnostic evaluation and treatment. Adv Clin Exp Med. 2016; 6: 1321-1330.

24 Lane DA, Kunz G, Olds RJ, et al. Molecular genetics of antithrombin deficiency. Blood Rev. 1996; 10: 59-74.

25 Bereczky Z, Kovács KB, Muszbek L. Protein C and protein S deficiencies: similarities and differences between two brothers playing in the same game. Clin Chem Lab Med. 2010; 48: 53-66.

26 de la Morena-Barrio M, Sandoval E, Llamas P, et al. High levels of latent antithrombin in plasma from patients with antithrombin deficiency. Thromb Haemost. 2017; 2017; 117: 880-888.

27 Alhenc-Gelas M, Gandrille S, Aubry ML, et al. Thirty-three novel mutations in the protein $\mathrm{C}$ gene. French INSERM network on molecular abnormalities responsible for protein C and protein S. Thromb Haemost. 2000 83: 86-92.

28 Alhenc Gelas M, Juin F, de Raucourt E, et al. Influence of PROS1 gene mutations affecting protein $S$ amino-acid 275 on plasma free protein $S$ measurement. Thromb Haemost. 2007; 97: 678-680.

29 de la Morena-Barrio ME, Sevivas TS, Martinez-Martinez I, et al. Congenital disorder of glycosylation (PMM2-CDG) in a patient with antithrombin deficiency and severe thrombophilia. J Thromb Haemost. 2012; 12 : 2625-2627.

30 Corral J, Huntington JA, González-Conejero $R$, et al. Mutations in the shutter region of antithrombin result in formation of disulfide-linked $\mathrm{d}$ mers and severe venous thrombosis. J Thromb Haemost. 2004; 6: 931-939.

31 Picard V, Dautzenberg MD, Villoutreix BO, et al. Antithrombin F229L: a new homozygous variant leading to spontaneous antithrombin polymerization in vivo associated with severe childhood thrombosis. Blood. 2003 102: $919-925$

32 Fitches AC, Lewandowski K, Olds RJ. Creation of an additional glycosylation site as a mechanism for type I antithrombin deficiency. Thromb Haemost. 2001; 4:1023-1027.

33 Tang L, Guo T, Yang R, et al. Genetic background analysis of protein $C$ deficiency demonstrates a recurrent mutation associated with venous thrombosis in Chinese population. PLoS One. 2012; 4: e35773.

34 Preston RJ, Ajzner E, Razzari $C$ et al. Multifunctional specificity of the protein C/activated protein C Gla domain. J Biol Chem. 2006; 39: 28850-28857.

35 Kuismanen K, Levo A, Vahtera E, et al. Genetic background of type protein C deficiency in Finland. Thromb Res. 2006; 5: 603-609.

36 Vincenot A, Gaussem P, Pittet JL, et al. Amino acids 225-235** of the protein $C$ serine-protease domain are important for the interaction with the thrombin-thrombomodulin complex. FEBS Lett. 1995; 2: 153-157.
Soria JM, Brito D, Barceló J, et al. Severe homozygous protein C deficiency: identification of a splice site missense mutation $(184,0->H)$ in exon 7 of the protein $C$ gene. Thromb Haemost. 1994; 1: 65-69.

38 Rovida E, Merati G, D'Ursi P, et al. Identification and computationally-based structural interpretation of naturally occurring variants of human protein C. Hum Mutat. 2007; 4: 345-355.

39 Wypasek E, Potaczek DP, Alhenc-Gelas M, et al. Novel missense mutation C106R in the PROC gene associated with type I protein $\mathrm{C}$ deficiency in a young Polish man with high-risk pulmonary embolism. Pol Arch Med Wewn. 2014; 1-2: 75-76.

40 Stenberg $Y$, Linse $S$, Drakenberg $T$, et al. The high affinity calciumbinding sites in the epidermal growth factor module region of vitamin K-dependent protein S. J Biol Chem. 1997; 37: 23255-23260.

41 van de Poel RH, Meijers JC, Bouma BN. The interaction between anticoagulant protein $\mathrm{S}$ and complement regulatory $\mathrm{C} 4 \mathrm{~b}$-binding protein (C4BP). Trends Cardiovasc Med. 2000; 2: 71-76.

42 Almasy L, Soria JM, Souto JC, et al. A quantitative trait locus influ encing free plasma protein $S$ levels on human chromosome 1q: results from henetic Analysis of Idiopathic Thrombophilia (GAIT) project. Arterioscle Thromb Vasc Biol. 2003; 23: 508-511.

43 Buil A, Soria JM, Souto JC, et al. Protein $\mathrm{C}$ levels are regulated by a quantitative trait locus on chromosome 16: results from the Genetic Analysis of Idiopathic Thrombophilia (GAIT) Project. Arterioscler Thromb Vasc Biol. 2004; 24: 1321-1325.

44 Medina P, Navarro S, Bonet E, et al. Functional analysis of two haplotypes of the human endothelial protein $\mathrm{C}$ receptor gene. Arterioscler Thromb Vasc Biol. 2014; 3: 684-690.

45 Kimura R, Kokubo Y, Miyashita K, et al. Polymorphisms in vitamin K-dependent gamma-carboxylation-related genes influence interindividual variability in plasma protein $\mathrm{C}$ and protein $\mathrm{S}$ activities in the general population. Int J Hematol. 2006; 84: 387-397.

46 Ungerstedt JS, Schulman S, Egberg N, et al. Discrepancy between antithrombin activity methods revealed in Antithrombin Stockholm: do fac tor Xa-based methods overestimate antithrombin activity in some patients? Blood. 2002; 6: 2271-2272.

47 Khor B, Van Cott EM. Laboratory tests for antithrombin deficiency. Am J Hematol. 2010; 85: 947-950. 